\title{
点集的伪径和布阵点数 *
}

路见可在文献 [1] 中证明了 $\mathbf{R}^{*}$ 中的有 界点集 $A$, 存在唯一的一个包含 $A$ 的最小球 体, 其中心称为 $A$ 的伪心, 其半径称为 $A$ 的伪 径, 并就 $A$ 是有限点集的情形, 描述性地给出 了求包含 $A$ 最小球体的方法. 我们把 这一 结果精确化, 得到了点集的直径和伪径相互 控制不等关系的最佳系数, 并用来给出布 阵点数控制上界的更好系数，显著改进了 Pollard ${ }^{[2]}$ 的结果.

我们的解决方法是从单纯形人手, 借助 欮知的 Helly 定理 ${ }^{[3]}$ 过渡到一般的有界点 集. 对单纯形的处理采用对维数的数学归纳 法, 主要解决外心在单纯形内部的情形。对 于外心在单纯形外的情形, 由于外接球体不 是包含单纯形的最小球体, 因此单纯形的顶 点并不全在包含它的最小球体的边界上, 可 以用降低维数的方法归结为外心在单纯形内 部的情形。基于引引理 1 , 这种降维的步嗓在整 个归纳过程中只是一开始使用一次, 往后就 畅通无阻了.

引理 $1 \mathbf{R}^{*}$ 中外心属于单纯 形这一 性质可保留到离外心最近的那个侧面的 $n-$ 1 维子单形上。

引理 2 设 $\mathrm{R}^{*}$ 中的单纯形 $G$ 包含了 它的外心, 则其内切球的半径 $r$, 以及外心至 各 $n-1$ 维子单形的最小距离 $r_{0}$ 不超过外 接球半径的 $\frac{1}{n}$.

定理 1 对 $\mathrm{R}^{*}$ 中的任何有界点集 $\boldsymbol{A}$, 其伪径 $\gamma(A)$ 和直径 $d(A)$ 满足

$$
\frac{1}{2} d(A) \leqslant r(A) \leqslant \sqrt{\frac{n}{2(n+1)} d(A),}
$$

第 17 期
两端的系数都不可改进.

作为应用，考虑 $\mathrm{R}^{*}$ 中直径为 $d$ 的有界 子集 $A$ 的 8 -布阵点数 (Packing number), 即从 $A$ 中挑出两两相距大于 8 的点所构成子 集的最大基数, 记作 $D(\varepsilon, A)$. Pollard 在 文献 [2] 中证明了

$$
D(\varepsilon, A) \leqslant\left(\frac{3 d}{\varepsilon}\right) .
$$

利用定理 1 , 可以改进为

定理 2

$$
D(\varepsilon, A) \leqslant\left(\sqrt{\frac{2 n}{n+1}} d+\varepsilon / \varepsilon\right) \text {. }
$$

当 $n=1$ 时, 这一结果已不可改进. 但 只要 $n \geqslant 2$, 仍有改进的余地. 因为证明时 所使用的小球填大球的方法不可避免留下空 隙,使估计精度降低。直观上,球体在相同直 径的点集中占据最大的地盘, 正单纯形顶点 布阵效率最高，据此对特例的精确计算使我 们猜测,理想的控制上界应该是

$$
D(\varepsilon, A) \leqslant \frac{2^{\frac{\pi}{2}-\left[\begin{array}{l}
n \\
2
\end{array}\right]\left[\begin{array}{l}
n \\
2
\end{array}\right]}}{n ! ! \sqrt{n+1}}\left(\frac{d}{8}+2\right)^{\bullet} .
$$

\section{* 文}

[1] 路见可,数学的实戟与认识, 1986,2: 69-73.

[2] Pollard, D., NSFCBMS Regional Conference Series in Prob. and Stat., Vol. 2, 1990.

[3] Brondsted, A., An Introdwction so Conbex Polyzopes, GTM 90, Springer-Verlsg, 1983.

陈培德

(中国科学院应用数学研究所, 北京100080)
- 国家自然利学基金资助项目

报 